\title{
Bayesian analysis of minimal model under the insulin-modified IVGTT
}

\author{
Yi Wang ${ }^{1}$, Kent M. Eskridge ${ }^{2}$, Andrzej T. Galecki ${ }^{3}$ \\ ${ }^{1,2}$ Department of Statistics, University of Nebraska-Lincoln, Nebraska, USA \\ ${ }^{3}$ Institute of Gerontology, University of Michigan, Michigan, USA
}

Received 24 September 2009; revised 23 October 2009; accepted 26 October 2009.

\begin{abstract}
A Bayesian analysis of the minimal model was proposed where both glucose and insulin were analyzed simultaneously under the insulin-modified intravenous glucose tolerance test (IVGTT). The resulting model was implemented with a nonlinear mixed-effects modeling setup using ordinary differential equations (ODEs), which leads to precise estimation of population parameters by separating the inter- and intra-individual variability. The results indicated that the Bayesian method applied to the glucose-insulin minimal model provided a satisfactory solution with accurate parameter estimates which were numerically stable since the Bayesian method did not require approximation by linearization.
\end{abstract}

Keywords: Minimal Model; Bayesian Analysis; IVGTT; Nonlinear Mixed-Effects Modeling; ODE

\section{INTRODUCTION}

Mixed-effects models that include both fixed and random effects to account for the inter-individual variability are becoming increasingly popular for analysis of population pharmacokinetic/pharmacodynamic (PK/PD) data [1-8, among others]. In these models it is assumed that all responses follow a similar functional form, but that parameters vary among individuals. By separating the interand intra-individual variability, mixed-effects models will often lead to more precise estimation of population parameters. Continuous pharmacokinetic processes are often described by systems of ordinary differential equations (ODE) which generally lead to models that are nonlinear in the parameters complicating estimation. However, closed form solutions for systems of differential equations are not always possible therefore numerical solutions of differential equations are necessary in order to deal with many types of population PK/PD problems. The nlmeODE in $\mathrm{R}$ given by Tornoe et al. [9] and
NLINMIX with ODE in SAS presented by Galecki et al. [10], which can handle the first-order ODE's by combining odesolve with the NLME and NLINMIX respectively, are used for parameter estimation in nonlinear mixed-effects models. In addition, WBDiff (WinBUGS Differential Interface) given by Lunn [11] is a useful tool for dealing with pharmacokinetic models defined by ODEs in the Bayesian setting. The analysis based on ODEs may offer practical benefits in terms of easier PK/PD modeling, particularly when more complicated mechanistic models are used [12].

Diabetes is associated with a large number of abnormalities in insulin metabolism, ranging from an absolute deficiency to a combination of deficiency and resistance, causing an inability to dispose glucose from the blood stream. Three factors: Insulin sensitivity, Glucose effectiveness, and Pancreatic responsiveness, referred to in Pacini and Bergman [13], play an important role for glucose disposal. Failure in any of these may lead to impaired glucose tolerance, or, if severe, diabetes. Quantitative assessment is possible by the minimal model [14], and may improve classification, prognosis and therapy of the disease. The minimal model is based on an intravenous glucose tolerance test (IVGTT), where glucose and insulin concentrations in plasma are sampled after an intravenous glucose injection. In patients with impaired glucose tolerant (IGT), the insulin response to glucose may be partially or totally suppressed. Of course, without the insulin response, the glucose disappearance model cannot provide an estimate of the metabolic parameters, since there is no input to the model. The insulin modification of IVGTT addressed the early problems with the minimal model 'failures' by insulin injection at $20 \mathrm{~min}$ utes after glucose injection is given at time zero. Traditionally, in the minimal model the glucose and insulin kinetics are described by two components, where the parameters traditionally have been estimated separately within each component by a nonlinear weighted least squares estimation technique in a two-step procedure [13]. The use of population analysis to extract all information, such as inter-subject variability, from experimental data 
brings about an improvement in the estimates of population and individual characteristics. Previous work with the IVGTT focused only on glucose kinetics where insulin is treated as a known with no measurement errors for non-Bayesian analysis [10] and for Bayesian analysis [15]. However, the glucose-insulin system is an integrated system and could be considered as a whole. An assumption of the minimal model is that glucose and insulin constitute a single dynamical system and important information is lost in treating the insulin as known. For example, pancreatic responsiveness, one important factor of the individual metabolic portrait, cannot be estimated if insulin is treated as known. Therefore, a Bayesian analysis was adopted in combination with population mixed-effects modeling to estimate the four population and individual metabolic indices simultaneously with the minimal model of glucose and insulin kinetics using data collected during insulin-modified intravenous glucose tolerance test (IVGTT). No other published work was identified that analyzed both glucose and insulin simultaneously under the insulin-modified IVGTT.

\section{METHODS}

\subsection{Bayesian Computational Algorithm for WBDiff}

Bayesian inference in WBDiff, which allows the numerical solution of arbitrary systems of ODEs within WinBUGS nonlinear mixed models, can be described based on the following hierarchical modeling.

1) Suppose a chemical or pharmacokinetic model is given by the systems of first-order ODE's with the form

$$
d x(t, \phi) / d t=g(x, t, \phi), x\left(t_{0}, \phi\right)=x_{0}(\phi), t \geq t_{0},
$$

where $x$ is an $k$-dimensional dependent variable vector, $g$ is the structural model while $\phi$ is a $q$-dimensional vector of unknown model parameters.

2) In nonlinear mixed-effects modeling, the withingroup variability describing the difference between the observed response value and the predicted value can be modeled as

$$
y_{i j} \sim N\left[E\left(y_{i j}\right)=f\left(x_{i j}, \varphi_{i}, t_{i j}\right) ; \sigma^{2}=\tau^{-1}\right]
$$

where $i=1, m$ subjects and $j=1, n_{i}$ time points; $X_{i j}$ is the solution of Eq.1 and the relationship between the observed response $y$ and the predicted variable $x$ is designated by a nonlinear function $f$.

3) The between-subject variability can be constructed by defining the subject-specific random effects as

$$
\phi_{i} \sim M V N_{p}(\theta, \Omega)
$$

where $\theta$ is a vector of mean population pharmacokinetic parameters and $\Omega$ is the variance-covariance matrix of between-subject random variability.

4) In addition, hierarchical modeling in a Bayesian setting comprises the prior specification, where prior distributions are assigned to $\tau, \theta$, and $\Omega$. For instance,

$$
\theta \sim M V N_{q}(\mu, \Sigma), \Omega^{1} \sim \operatorname{Wishart}_{p}(R, \rho), \tau \sim \operatorname{Gamma}(a, b)(4)
$$

The Bayesian inference is based on the following principle: posterior $\propto$ prior $\mathrm{x}$ likelihood. That is, the so-called "likelihood function" is used to update "prior beliefs" about some unknown parameters of interest to "posterior beliefs" in the light of observed data. To obtain the posterior estimators, using Monte Carlo approximation we simulate values from the joint posterior distribution of all the model parameters given the observed data, more specifically, the full conditional distribution. For example, the logarithm of the full conditional distribution for the random effect $\phi_{1}$ can be constructed as follows:

$$
\begin{gathered}
\log f\left(\phi_{i} \mid \cdot\right) \propto \log f\left(\phi_{i} \mid \theta, \Omega\right)+\sum_{i=1}^{m} \sum_{j=1}^{n_{i}} \log f\left(y_{i j} \mid \phi_{i}, \tau\right) \\
\propto-\frac{1}{2} \log |\Omega|-\frac{1}{2}\left(\phi_{i}-\theta\right)^{T} \Omega^{-1}\left(\phi_{i}-\theta\right)+ \\
\frac{n}{2} \log \tau-\frac{1}{2} \sum_{i=1}^{m} \sum_{j=1}^{n_{i}}\left(y_{i j}-f\left(x_{i j}, \phi_{i}, t_{i j}\right)\right)^{2} \tau
\end{gathered}
$$

where the dot notation in $f\left(\phi_{i} \mid \cdot\right)$ denotes the distribution of $\phi_{1}$ conditional upon everything else in the model. The term $\log f\left(\phi_{i} \mid \theta, \Omega\right)$ refers to the logarithm of the prior distribution for $\phi_{1}$ and this is specified to be a multivariate normal distribution with mean vector $\theta$ and variance -covariance matrix $\Omega$. The term $\log f\left(y_{i j} \mid \phi_{i}, \tau\right)$ refers to the log-likelihood of the $j^{\text {th }}$ observation for subject $i$ under the model, and the concentration $y_{i j}$ was assumed to follow a normal distribution with mean $f\left(x_{i j}, \phi_{i}, t_{i j}\right)$ and variance $\tau^{-1}$. Since we do not have a closed form for $x_{i j}$ from Eq.1, the numerical solution $x_{i j}$ has to be obtained from Eq.1 by fixing all the conditioning parameters so that the Gibbs sampler can generate a new value, say $\phi_{1}^{(1)}$ from the full conditional distribution (Eq.5) given the initial values to each unknown parameters $\theta^{(0)}, \Omega^{(0)}$, and $\tau^{(0)}$. After $n$ such iterations, the algorithm yields a joint sample $\phi_{1}^{(n)}$, which can be used for statistical inference in WBDiff. The full conditional distributions for the other model parameters can be constructed in a similar manner.

\subsection{Population Analysis on Glucose-Insulin Minimal Model}

In this section, Bayesian analysis in combination with population mixed-effects modeling was used to simultaneously estimate the four population and individual metabolic indices: insulin sensitivity $\left(S_{\mathrm{I}}\right)$, glucose effectiveness $\left(S_{\mathrm{G}}\right)$ and pancreatic responsiveness ( $\phi_{1}$ and $\left.\phi_{2}\right)$, based on the integrated glucose-insulin minimal model 
using data collected during the insulin-modified intravenous glucose tolerance test (IVGTT).

\subsubsection{Bergman's Modified Minimal Model}

In an IVGTT study a dose of glucose was administered intravenously over a 60 seconds period to overnightfasted subjects, 20 min after the glucose bolus, insulin was injected over 1-2 min either into the portal vein or into the femoral vein, and subsequently the glucose and insulin concentrations in plasma were frequently sampled (usually 30 times) over a period of 180 minutes. Profiles of the 10 patients are displayed in Figure 1 [10].

Based on Figure 1, the intravenous glucose dose immediately elevates the glucose concentration in the plasma forcing the pancreatic $\beta$-cells to secrete insulin. The insulin in the plasma is hereby increased, and the glucose uptake in muscles, liver and tissue is raised by the remote insulin in action. This lowers the glucose concentration in plasma, implying the $\beta$-cells to secrete less insulin, from which a feedback effect arises [16]. The integrated glucose-insulin system can be described by the following non-linearly coupled system of differential equations, but this approach is not exactly the same model as used in [17]. Here $I_{1}$ is introduced to account for the injection of insulin at $t_{1}$ after the glucose bolus during the IVGTT:

$$
\begin{array}{cc}
d G / d t=-p_{1}\left(G(t)-G_{b}\right)-X(t) G(t), & G(0)=G_{0}, \\
d X / d t=-p_{2} X(t)+p_{3}\left(I(t)-I_{b}\right), & X(0)=0, \\
d I / d t=-n\left(I(t)-I_{b}\right)+\gamma(G(t)-h)^{+} t, & I(0)=I_{0}, I\left(t_{1}\right)=I_{1},
\end{array}
$$

where $t=0$ is the glucose injection time, + denotes positive reflection, namely,

$$
(G(t)-h)^{+}=\left\{\begin{array}{cc}
G(t)-h, & G(t)>h, \\
0, & \text { otherwise. }
\end{array}\right.
$$

and the model parameters are as explained in [13].

\subsubsection{Bayesian Analysis of Bergman's Modified Minimal Model Using WBDiff}

A Bayesian framework for modeling the time-varying glucose and insulin profiles during the IVGTT and inter-individual variability requires a three-stage hierarchical model. At the first stage, glucose values $G\left(t_{\mathrm{j}}, \theta_{\mathrm{i}}\right)$ and insulin values $I\left(t_{\mathrm{j}}, \theta_{\mathrm{i}}\right)$ in subject $i$ at time $t_{\mathrm{j}}$ were obtained as the solutions to Eq.6. The model we consider assumes $G_{\mathrm{ij}}=G\left(t_{\mathrm{j}}, \theta_{\mathrm{i}}\right)+\varepsilon_{\mathrm{ij} 1}$ and $I_{\mathrm{ij}}=I\left(t_{\mathrm{j}}, \theta_{\mathrm{i}}\right)+\varepsilon_{\mathrm{ij} 2}$, where $\varepsilon_{\mathrm{ijk}}$ is a mean zero normally and independently distributed error term. An additional assumption about withinsubject errors will be forthcoming in due course. Stacking the two response variables $G_{\mathrm{ij}}$ and $I_{\mathrm{ij}}$ into a single response vector, an indicator variable for the two responses can be used to construct the model function. By combin- ing $G(\cdot)$ and $I(\cdot)$, we obtain

where $Y_{i j}=\left(G_{i j}, I_{i j}\right)^{T}, \quad f_{k}\left(t_{j}, \theta_{i}\right)=\left\{\begin{array}{ll}G\left(t_{j}, \theta_{i}\right), & k=1 \\ I\left(t_{j}, \theta_{i}\right), & k=2\end{array}\right.$ and $\varepsilon_{i j}=\left(\varepsilon_{i j 1}, \varepsilon_{i j 2}\right)^{T} . \theta_{\mathrm{i}}$ is a vector of parameters of this model for subject $i$ denoted by

$$
\theta_{i}=\left(p_{1 i}, p_{2 i}, p_{3 i}, G_{0 i}, n_{i}, \gamma_{i}, h_{i}, I_{0 i}, t_{1 i}, I_{1 i}\right)^{T} .
$$

Here it is further assumed that the time when insulin injection occurs after glucose injection at time zero is also an unknown parameter denoted as $t_{1}$ which must be estimated. To account for correlation between the two response variables measured on the same occasion, we may assume the elements of $\varepsilon_{\mathrm{ij}}$ are correlated, with variance-covariance matrix $\tau^{-1} \Lambda_{2}$. Here for simplicity it is assumed that $\Lambda_{2}=I_{2}$, since the intent in this paper is to introduce the random effects to account for inter-individual variability rather than define a variance-covariance structure for random errors to address intra-individual variability. However, the variance function $f_{\mathrm{k}}^{2}\left(t_{\mathrm{j}}, \theta\right)$ and the weight $w_{\mathrm{j}}$ are specified for heterogeneous within-subject error $\left(\varepsilon_{\mathrm{ijk}}\right)$ variance for two reasons: 1) the glucose and insulin concentration points before 8 minutes can then be zero-weighted to account for the time taken by the injected glucose to diffuse in its distribution space, which can be achieved by setting $w_{\mathrm{j}}=0$ at any time points before 8 minutes and $w_{\mathrm{j}}=1$ for others; 2) it is commonly recognized that intra-subject variation of this kind tends to increase with plasma concentration level. That is, the higher the concentration level, the larger the variation so that less weight should be assigned. Thus, we assume the following covariance matrix for the within-subject errors $\varepsilon_{\mathrm{ijk}}$.

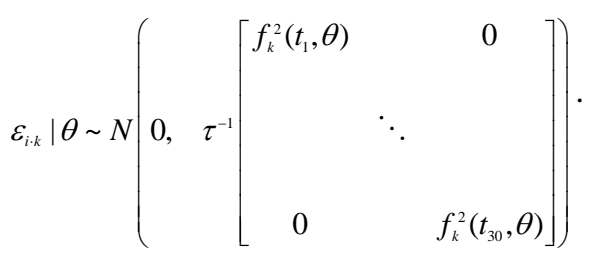

The second stage is characterized by making assumptions about individual parameters. In particular, it was assumed that the individual parameters were drawn from a multivariate log-normal distribution guaranteeing nonnegativity of parameters

$$
\left(p_{1 i}, p_{2 i}, p_{3 i}, G_{0 i}, n_{i}, \gamma_{i}, h_{i}, I_{0 i}, t_{1 i}, I_{1 i}\right)^{T} \sim \operatorname{Lnormal}\left(\mu, \Sigma_{10 \times 10}\right),
$$

where $\mu$ is an unknown population mean vector and $\Sigma$ is an unknown covariance matrix. At the third stage, prior distributions for population parameters $\mu, \Sigma$, and $\tau$ were specified. These prior distributions were vague representing a 'lack' of prior knowledge with: 
GLUCOSE(mg/dl)

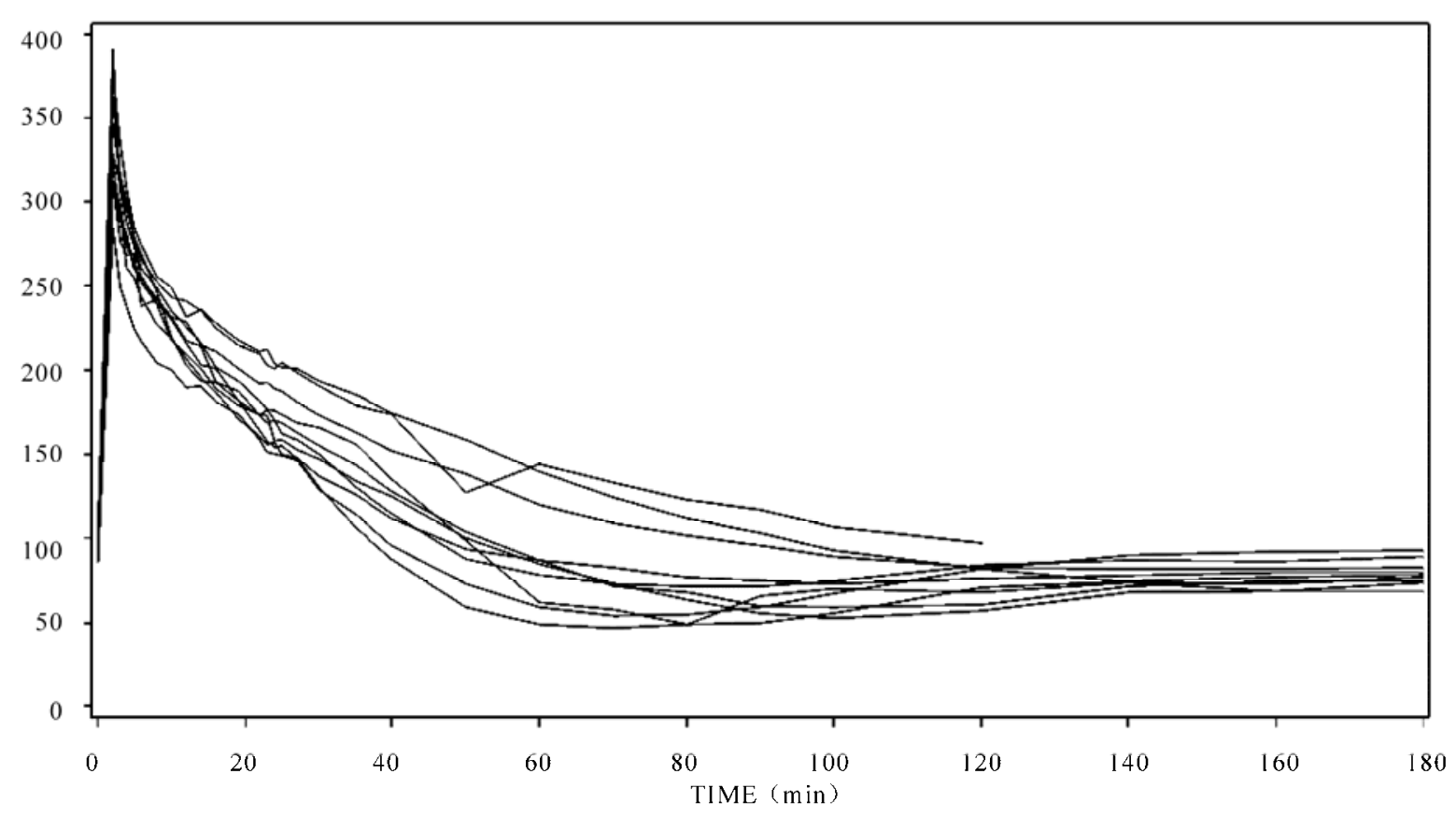

$\operatorname{INSULIN}(\mathrm{mU} / \mathrm{l})$

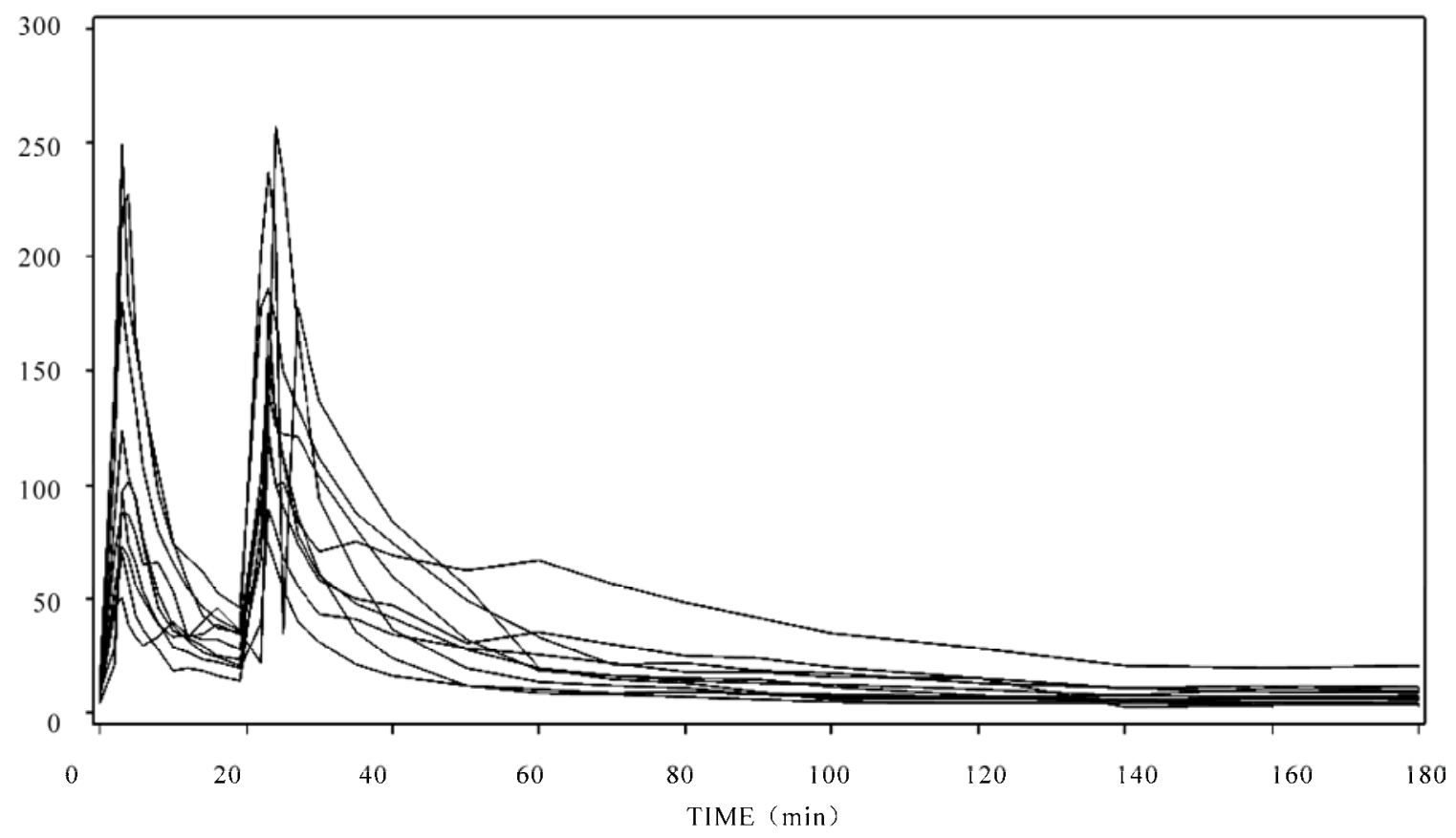

Figure 1. Individual glucose and insulin profiles for 10 patients.

$\mu \sim \operatorname{Normal}\left[\hat{\mu}, 10^{4} I_{10}\right], \quad \Sigma^{-1} \sim$ Wishart $\left[10,0.01 I_{10}\right]$, $\tau \sim \operatorname{Gamma}(0.001,0.001)$,

where

$$
\hat{\mu}=(-3.5,-4.6,-10,-1.35,-5.8,4.4,5.6,5.9,5.8,3)^{T}
$$

was the mean for $\mu$ derived from nonlinear weighted least squares minimization on

$$
\sum_{i=1}^{10} \sum_{j=1}^{t_{i}} w_{i j}\left\{\left(G_{i j}-G\left(t_{j}, \theta_{i}\right)\right)^{2}+\left(I_{i j}-I\left(t_{j}, \theta_{i}\right)\right)^{2}\right\} \cdot
$$


In order to allow the experimental data to drive the estimation process, the above prior distributions specified virtually 'flat' distributions, i.e. they indicated that all values occurred with nearly the same probability, although informative prior distributions could be used as there is a wealth of information about parameters of the minimal model in various populations. A general discussion about the form of vague prior distribution can be found in [18]. Note that the priors specified above implements the common assumption that population parameters are not correlated, but allows the posterior estimates to demonstrate correlation.

\section{RESULTS}

For the calculations, we employed WBDiff which incorporates the numerical solution of ODEs into the WinBUGS program. The WinBUGS program adopted the Metropolis-Hastings algorithm to calculate a single chain with 15,000 samples, from which the first 5,000 samples were discarded and the remaining 10,000 samples were used in a further analysis. The Bayesian analysis provided the following population parameter estimates and the posterior distribution of the parameters $\mu$ in log scale, $\Sigma$, and $\tau$ summarized by the median, the mean and the $95 \%$ credible interval respectively presented in Table $\mathbf{1}$.

The chain history was stable, showing the classic "fuzzy caterpillar" shape, with minimal evidence of autocorrelation in the samples generated from the posterior distribution. After observing the fitted plots (Figure 2), the fitted model sufficiently explained the kinetics of glucose and insulin, since the observed and predicted values matched reasonably well except for several observations ignored since during the first eight minutes after injection, at the early time points. Those early data points were the pattern of plasma glucose and insulin is dominated by extra cellular mixing. The estimates of the fixed-effects parameters were also satisfactory with an acceptable precision (range of coefficient of variation 1.29-8.78\%) and within the normal range. For example, the time at which the insulin is injected should be between 20 and $22 \mathrm{~min}$, and our estimate $t_{1}$ is $\exp (3.031)=20.72 \mathrm{~min}$. It was also possible to determine the individual estimates of parameters by examining the behavior of

Table 1. Bayesian parameter estimates and 95\% credible intervals.

\begin{tabular}{|c|c|c|c|c|c|c|c|}
\hline Node & Mean & SD & MC error & $2.50 \%$ & Median & $97.50 \%$ & Sample \\
\hline$p_{1}$ & -3.714 & 0.185 & 0.016 & -4.095 & -3.693 & -3.399 & 10000 \\
\hline$p_{2}$ & -5.096 & 0.448 & 0.043 & -5.878 & -5.081 & -4.315 & 10000 \\
\hline$p_{3}$ & -12.24 & 0.171 & 0.015 & -12.53 & -12.26 & -11.87 & 10000 \\
\hline$n$ & -2.026 & 0.102 & 0.007 & -2.244 & -2.021 & -1.837 & 10000 \\
\hline$\gamma$ & -7.08 & 0.166 & 0.013 & -7.427 & -7.071 & -6.778 & 10000 \\
\hline$h$ & 4.264 & 0.07 & 0.003 & 4.138 & 4.26 & 4.413 & 10000 \\
\hline$G_{0}$ & 5.529 & 0.072 & 0.003 & 5.389 & 5.53 & 5.672 & 10000 \\
\hline$I_{0}$ & 4.804 & 0.109 & 0.007 & 4.574 & 4.808 & 5.01 & 10000 \\
\hline$I_{1}$ & 4.958 & 0.108 & 0.006 & 4.743 & 4.961 & 5.162 & 10000 \\
\hline$t_{1}$ & 3.031 & 0.059 & 0.002 & 2.906 & 3.033 & 3.141 & 10000 \\
\hline$\sigma_{\mathrm{p} 1}^{2}$ & 0.211 & 0.177 & 0.008 & 0.05 & 0.164 & 0.65 & 10000 \\
\hline$\sigma_{\mathrm{p} 2}^{2}$ & 0.136 & 0.117 & 0.006 & 0.027 & 0.104 & 0.439 & 10000 \\
\hline$\sigma_{\mathrm{p} 3}^{2}$ & 0.241 & 0.322 & 0.022 & 0.026 & 0.124 & 1.132 & 10000 \\
\hline$\sigma_{\mathrm{n}}^{2}$ & 0.067 & 0.079 & 0.005 & 0.008 & 0.044 & 0.28 & 10000 \\
\hline$\sigma_{\gamma}^{2}$ & 0.03 & 0.036 & 0.002 & 0.002 & 0.02 & 0.117 & 10000 \\
\hline$\sigma_{h}^{2}$ & 0.012 & 0.015 & 0.001 & 0.001 & 0.007 & 0.051 & 10000 \\
\hline$\sigma_{\mathrm{G} 0}^{2}$ & 0.089 & 0.082 & 0.003 & 0.017 & 0.067 & 0.295 & 10000 \\
\hline$\sigma_{\mathrm{I} 0}^{2}$ & 0.059 & 0.045 & 0.002 & 0.013 & 0.047 & 0.177 & 10000 \\
\hline$\sigma_{\mathrm{I} 1}^{2}$ & 0.297 & 0.262 & 0.015 & 0.068 & 0.22 & 0.981 & 10000 \\
\hline$\sigma_{\mathrm{t} 1}^{2}$ & 0.867 & 0.907 & 0.054 & 0.131 & 0.615 & 3.137 & 10000 \\
\hline$\tau^{-1}$ & 0.018 & 0.001 & 0 & 0.015 & 0.018 & 0.02 & 10000 \\
\hline
\end{tabular}



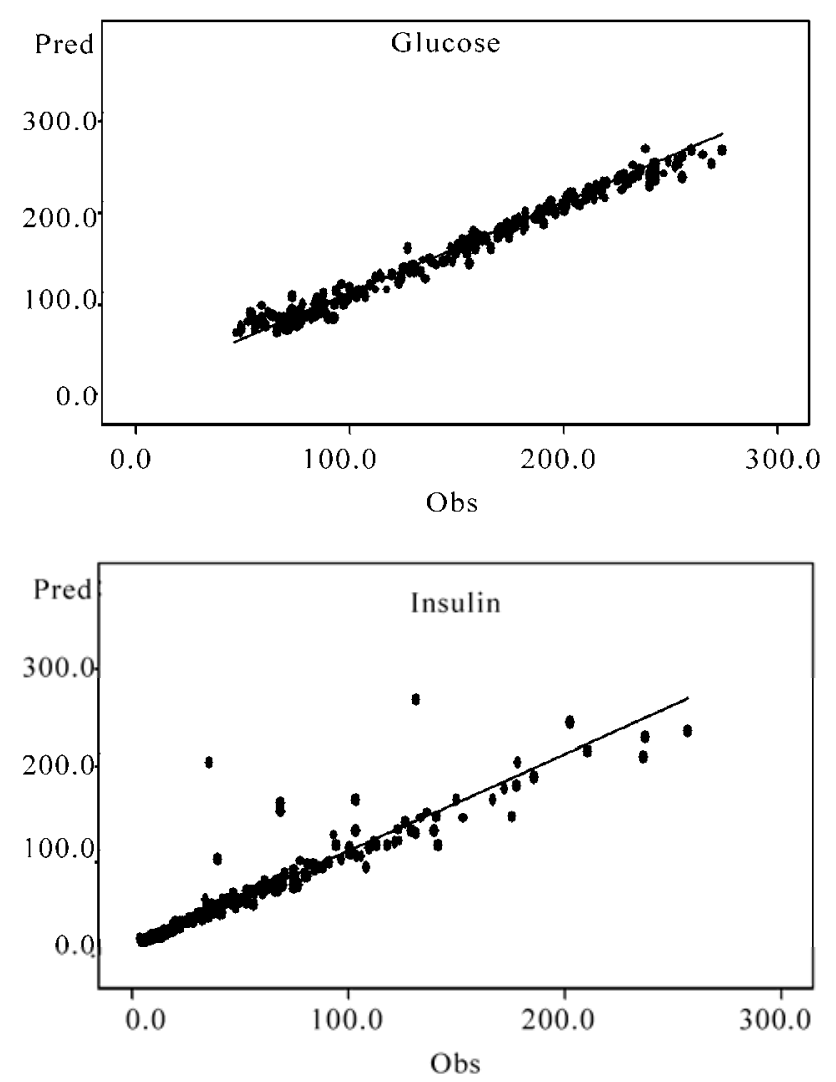

Figure 2. Model fit plots between predicted (Pred) and observed (Obs) concentrations under WBDiff. The upper plot describes the fit of glucose kinetics and the lower plot described the fit of insulin kinetics.

$$
\left(p_{1 i}, p_{2 i}, p_{3 i}, G_{0 i}, n_{i}, \gamma_{i}, h_{i}, I_{0 i}, t_{1 i}, I_{1 i}\right)^{T}
$$

for each subject $i$. It was also possible to calculate the group and individual profiles based on the above parameter estimates. Specifically, the population characteristics of the minimal model for the data set used here were: Insulin sensitivity:

$$
S_{\mathrm{I}}=p_{3} / p_{2}=\exp (-12.24) / \exp (-5.096)=7.9 \times 10^{-4} ;
$$

Glucose effectiveness: $S_{\mathrm{G}}=p_{1}=2.44 \times 10^{-2}$; Pancreatic responsiveness: $\phi_{2}=\gamma \times 10^{4}=8.42$ where $\phi_{1}$ can be calculated for each subject.

\section{DISCUSSIONS}

With the traditional minimal model, the kinetic parameters of the two components, glucose and insulin, are estimated separately using weighted nonlinear least squares within each component. In this work, the two components are combined to obtain a unified, integrated glucoseinsulin system so that four metabolic indices: $S_{\mathrm{I}}, S_{\mathrm{G}}, \phi_{1}$ and $\phi_{2}$, which represent an integrated metabolic portrait of a single individual, can be estimated simultaneously during the insulin-modified IVGTT. The integrated analysis can be directly applied to the protocols without insulin modification. Under insulin-modified IVGTT, not only the glucose kinetics but also the insulin kinetics can be fitted in a satisfactory way based on the Bayesian hierarchical analysis by introducing $I_{1}$ and $t_{1}$ in the minimal model and estimating them together with other model parameters. This approach constitutes an attractive option for minimal model analyses, since in most of the literature, the converse model of pancreatic secretion cannot be fitted to the observed data with the use of pharmacologic agents, resulting in the focus of most previous work on the glucose kinetics regarding insulin as the input to the system $[10,13,15]$.

In addition, it is important to note that the nonBayesian population analysis with the required linearization approximation cannot be applied to the insulin system alone and glucose-insulin kinetics in the proper way, since linearization itself restricts its application with this particular PK/PD model. In the minimal model Eq.6, $\max (G(t)-h, 0)$ is not differentiable with respect to $h$ at the time points when $G(t)=h$. In order to make the integrand function $\frac{\partial \dot{I}(t)}{\partial h}$ well defined, the non-differentiable points must be specified as intermediate points, and then $\frac{\partial \dot{I}(t)}{\partial h}$ must be defined over the subintervals with non-differentiable points as endpoints, so that the numerical solution of $\frac{\partial I}{\partial h}$ can be solved from 'ode' module. But, the question remains as to where the non-differentiable time points are located in addition to the fact that for different subjects they do not necessarily coincide and may vary from iteration to iteration due to the change of the estimate of $h$ during optimization. These appear to be serious problems that cannot be overcome when using a non-Bayesian approach with the required linearization. Another potential limitation of linearization is revealed when nonlinearity curvature in the parameter effects is severe due to the complexity of Eq.6. One naive approach is to solve the problem with non-differentiable sensitivity by replacing $\max (G(t)-h, 0)$ by $G(t)-h$ in the minimal model equations. We fitted this modified model to the data, however, not too many improvements have been obtained when compared with non-Bayesian method applied to Eq.6. In fact, it is sensible to specify $\max (G(t)-h, 0)$ in Eq.6, since insulin enters the plasma with zero rate when glucose in plasma is below the threshold amount.

Overall, for the glucose-insulin minimal model, the Bayesian approach appears to be the preferred since the algorithm behind the Bayesian approach is applicable and effective to the structure of minimal model, and sensitivities are not needed in the estimation process as with the non-Bayesian approach. Another advantage of Bayesian analysis is that individual estimates of model parameters can be simultaneously calculated under the 
Bayesian process. Therefore, Bayesian appro ach should be considered as an additional tool for data analysis, since it enables the analysis of systems of ODEs by nonlinear mixed-effects modeling and provide precise parameter estimates, make them useful tools for population PK/PD analysis of complicated systems of ODEs with and without an explicit form solution.

\section{ACKNOWLEDGEMENTS}

We gratefully acknowledge support from the Claude Pepper Center Grants AG08808 and AG024824 from the National Institute of Aging.

\section{REFERENCES}

[1] Beal, S.L. and Sheiner, L.B. (1982) Estimating population kinetics. CRC Critical Reviews in Bioengineering, 8, 195-222.

[2] Lindstrom, M.J. and Bates, D.M. (1990) Nonlinear mixed effects models for repeated measures data. Biometrics, $\mathbf{4 6}$, 673-687.

[3] Lindsey, J.K. (1993) Models for repeated measurements. Oxford.

[4] Davidian, M. and Giltinan, D.M. (1995) Nonlinear models for repeated measurement data. Chapman and Hall.

[5] Vonesh, E.F. and Chinchilli, V.M. (1996) Linear and nonlinear models for the analysis of repeated measurements. Marcel Dekker.

[6] Pinheiro, J.C. and Bates, D.M. (2000) Mixed-effects models in S and Splus. Springer.

[7] Diggle, P.J., Heagerty, P., Liang, K.Y. and Zeger, S.L. (2002) Analysis of longitudinal data. Oxford.

[8] Demidenko, E. (2004) Mixed models: Theory and applications. John Wiley \& Sons, Canada.

[9] Tornoe, C.W., Agerso, H., Jonsson, E.N., Madsen, H. and
Nielsen, H.A. (2004) Non-linear mixed-effects pharmacokinetic/pharmacodynamic modeling in NLME using differential equations. Computer Methods and Programs in Biomedicine, 76, 31-40.

[10] Galecki, A.T., Wolfinger, R.D., Linares, O.A., Smith, M.J. and Halter, J.B. (2004) Ordinary differential equation PK/PD using the SAS macro NLINMIX. Journal of Biopharmaceutical Statistics, 14(2), 483-503.

[11] Lunn, D.J. (2004) WinBUGS differential interfaceworked examples. www.winbugs-development.org.uk.

[12] Wang, Y., Eskridge, K.M. and Zhang, S. (2008) Semiparametric mixed-effects analysis of PK/PD models using differential equations. Journal Pharmacokinetics and Pharmacodynamics, 35, 443-463.

[13] Pacini, G. and Bergman, R.N. (1986) MINMOD: A computer program to calculate insulin sensitivity and pancreatic responsivity from the frequently sampled intravenous glucose tolerance test. Computer Methods and Programs in Biomedicine, 23, 113-122.

[14] Bergman, R.N., Ider, Y.Z., Bowden, C.R. and Cobelli, C. (1979) Quantitative estimation of insulin sensitivity. American Journal of Physiology, 236, E667-E677.

[15] Agbaje, O.F., Luzio, S.D., Albarrak, A.I.S., Lunn, D.J., Owens, D.R. and Hovorka, R. (2003) Bayesian hierarchical approach to estimate insulin sensitivity by minimal model. Clinical Science, 105, 551-560.

[16] Topp, B., Promislow, K. and De Vries, G. (2000) A model of $\beta$-cell mass, insulin, and glucose kinetics: Pathway to diabetes. Journal of Theoretical Biology, 206, 615-619.

[17] De Gaetano, A. and Arino, O. (2000) Mathematical modeling of the intravenous glucose tolerance test. Journal of Mathematical Biology, 40(2), 136-168.

[18] Gamerman, D. (1997) Markov chain monte carlo: Stochastic simulation for bayesian inference. Chapman \& Hall. 